\title{
Ferroelectric Research at the University of Washington During the Period 1955-70
}

\section{Authors: Edwin A. Uehling, John L. Bjorkstam, and V. Hugo Schmidt}

This is an Accepted Manuscript of an article published in Ferroelectrics in August 1987, available online: http://www.tandfonline.com/10.1080/00150198708201309.

Uehling, Edwin A., John L. Bjorkstam, and V. Hugo Schmidt. "Ferroelectric Research at the University of Washington During the Period 1955-70." Ferroelectrics 74, no. 1 (August 1987): 293-300. doi: 10.1080/00150198708201309. 


\title{
FERROELECTRIC RESEARCH AT THE UNIVERSITY OF WASHINGTON DURING THE PERIOD 1955-70 $\dagger$
}

\author{
EDWIN A. UEHLING $\ddagger$ \\ Department of Physics, University of Washington, Seattle, Washington USA \\ JOHN L. BJORKSTAM \\ Department of Electrical Engineering, University of Washington, Seattle, \\ Washington USA \\ V. HUGO SCHMIDT \\ Department of Physics, Montana State University, Bozeman, Montana USA
}

(Received May 3, 1985)

Major accomplishments in ferroelectric research during the period 1955-70 stimulated in whole or in part by work initiated at the UW include: (a) the first observation of a deuteron magnetic resonance in a hydrogen-bonded solid, thus opening up a new technique for studying hydrogen bond dynamics; (b) theoretical and experimental exploitation of a model of hydrogen bond dynamics which takes account of both interbond and intrabond motion; (c) a satisfactory resolution of major theoretical problems associated with phase changes in hydrogen bonded ferroelectrics; and (d) reinterpretation of phase transition phenomena through the successful application of experimental techniques different from nuclear magnetic resonance such as electrical conductivity, domain dynamics, and velocity of sound measurements.

In retrospect it would appear that two factors made important contributions to the initiation of the research and to the direction which it took. The first was the work of Warren Proctor and a student, Walter Tanttila, at the UW on the saturation of nuclear quadrupole energy levels in $\mathrm{NaClO}_{3}$ by ultrasonic excitation. It was this work which stimulated our interest in nuclear magnetic relaxation and was to prove useful later on. The second factor is of a more serendipitous nature. Present at the time was a graduate student, John Bjorkstam, who was just at the stage of starting his research, but not yet with an assigned problem. He recalls that, as a student in a solid state physics course taught by Edwin Uehling, his interest was stimulated by the fact that the ferroelectric transition temperature in certain hydrogen bonded crystals is strongly influenced by deuteration. On the basis of this observation alone it had already been noted many times that the hydrogen bond must play a central role in the determination of ferroelectric

$†$ Paper 3 in an ongoing series on history of ferroelectricity.

$\ddagger$ Recently deceased. 
behavior in these crystals. But only the vaguest notions of the relevant mechanism had been expressed up to this time and very little experimental data were available. It was Bjorkstam's continuing interest in this problem which led first to the necessary acquisition of deuterated crystals, not previously available in this laboratory, and then to the long and ultimately successful search for the deuteron resonance. The first report on finding the resonance was at the Stanford APS meeting in $1957 . .^{1}$ The observation was made on a $1 \mathrm{~cm}^{3}$ single crystal of $\mathrm{KD}_{2} \mathrm{PO}_{4}{ }^{2}$ using a continuous wave (cw) Pound-Knight marginal oscillator operating at very low rf level in conjunction with a 7 inch pole diameter permanent magnet which placed the resonance for deuterons near $8 \mathrm{MHz}$. Some results on spin-lattice relaxation time $T_{1}$, and domain effects, were also reported at this meeting.

During the next couple of years Bjorkstam made a study of both the deuterated and non-deuterated versions of $\mathrm{KH}_{2} \mathrm{PO}_{4}$ type crystals. The study of the deuterated crystal produced detailed quantitative information on the electric field gradient (efg) tensor at the hydrogen site, the thermal relaxation time $T_{1}$ as a function of frequency, crystal orientation, and temperature over a temperature range extending from room temperature to below the Curie point $T_{c}$, and the resonance line width. An observed extra splitting in the deuteron resonance below $T_{c}$ was consistent with the existence of domain effects. These results are reported in Bjorkstam's thesis ${ }^{3}$ and in a subsequent paper which includes a provisional theoretical analysis of the thermal relaxation data. ${ }^{4}$

A persistent problem throughout this study was that of finding a suitable basis for a theory of relaxation which would explain the dependence of deuteron $T_{1}$ on temperature, frequency, and crystal orientation. Two contributions to relaxation were under consideration at this time: (1) dissipation of spin energy to paramagnetic impurities; and, (2) interaction of the nuclear quadrupole moment with a thermally induced time varying electric field gradient due to lattice vibrations and interbond jumping. The first of these two contributions was studied using as probes the protons of $\mathrm{KH}_{2} \mathrm{PO}_{4}$ and $\mathrm{KH}_{2} \mathrm{AsO}_{4}$ and the phosphorus nuclei of $\mathrm{KH}_{2} \mathrm{PO}_{4}$. While a precise value for the paramagnetic ion contribution to deuteron relaxation was not extracted from experiments such as these until later, the results obtained showed that the contribution would be small, but significant. ${ }^{3,5}$ Its magnitude would also be relatively insensitive to temperature, frequency, and crystal orientation. Hence, it was not unreasonable to neglect it completely in a first attempt to test another model of relaxation which might be presumed to be dominant. The model selected was based on the stimulation of spin transitions by random fluctuations in the efg tensor due to interbond jumping, the only unknown parameter being the correlation time associated with the jump motion. It was found ${ }^{4}$ that some features of the experimental results were satisfactorily described, but the model is deficient in several significant ways, one of which is an incorrect description of the $T_{1}$ angular dependence.

Before moving to the second phase of this research mention should be made of one other observation in this earlier period while studying proton relaxation in $\mathrm{KH}_{2} \mathrm{AsO}_{4}$. It was found that the proton $T_{1}$ decreases sharply as the temperature is lowered through the Curie point. This is not observed in any other isomorph and 
is clearly due to the presence of arsenic. An explanation in terms of crossrelaxation was given several years later by Bjorkstam. ${ }^{6}$ A demonstration of the precision with which cross-over data can be duplicated by a calculation based on crystal structure and the measured efg tensor was then given by Beezhold. ${ }^{7}$

It is now Autumn 1958. Bjorkstam has taken his Ph.D. examination and has become a full time member of the Faculty in the UW Department of Electrical Engineering where after only two years he will be promoted to Associate Professor and eventually of course to Professor. He is immediately successful in the establishment of an independent research laboratory well staffed after a few years with competent research students. A program of research covering a wide spectrum in ferroelectrics is developed. These include: (1) continuing deuteron NMR in hydrogen bonded materials; ${ }^{8}$ (2) proton and ${ }^{75} \mathrm{As} \mathrm{NMR}$ in $\mathrm{KH}_{2} \mathrm{AsO}_{4}$ type crystals; ${ }^{9}(3)$ domain dynamics and polarization studies in Rochelle salt and $\mathrm{KD}_{2} \mathrm{PO}_{4}$ type crystals, ${ }^{10}$ (4) EPR in $\mathrm{BaTiO}_{3} ;{ }^{11}(5)$ optical harmonic generation in $\mathrm{KD}_{2} \mathrm{PO}_{4} ;{ }^{12}$ and, (6) dielectric relaxation. ${ }^{13}$ From the beginning a healthy spirit of collaboration between the two groups located only 2000 feet apart on the same university campus has contributed to the research of both groups and continues to the present day.

Meanwhile V. Hugo Schmidt has been preparing himself to take over from Bjorkstam who had previously given some guidance in Schmidt's indoctrination into the techniques of nuclear magnetic resonance. An important turning point at this stage in the study of deuteron relaxation was the earlier observation made by Bjorkstam, however without follow-up until now, of the fact that the saturation of say an $\mathrm{X}$-bond resonance in $\mathrm{KD}_{2} \mathrm{PO}_{4}$ disturbed the level populations observed subsequently in a Y-bond resonance. Two mechanisms capable of producing such effects which come immediately to mind are cross-relaxation and the physical exchange of positions. To choose between them Schmidt constructed a superheterodyne pulse NMR crossed-coil transmitter-receiver apparatus and conclusively demonstrated that the deuterons did in fact exchange their positions between $\mathrm{X}$ and $\mathrm{Y}$ bonds. Such exchanges in a three-dimensional lattice should be associated with ionic conductivity, as was pointed out by Onsager. ${ }^{14}$ By making conductivity and $T_{1}$ measurements, Schmidt showed that such ionic conduction is the mechanism for electrical conductivity in this crystal. The proof was in the magnitude of the activation energy which is the same for conductivity and $T_{1}$ measurements and equal to $0.58 \mathrm{eV}$. To separate the interbond contribution to relaxation from other contributions it was necessary to go to low field. Because of the long $T_{1}$, of the order of 10 minutes, it was possible to do this by saturating the spin system, going to low field by simply turning down the field, and then after the desired waiting time going back to high field and measuring the signal recovery.

Onsager also pointed out the possibility of intrabond deuteron motion, ${ }^{15}$ and this suggestion helped unravel the various contributions to the spin-lattice relaxation. As previously mentioned, Bjorkstam had already made some orientation-dependent measurements of $T_{1}$ at room temperature, but, unfortunately, three relaxation mechanisms, namely, interbond jumps, intrabond jumps, and spin diffusion to paramagnetic impurities, all make comparable contributions at room temperature and at the frequency $(7.5 \mathrm{MHz})$ of observation. As 
mentioned above, the slow interbond jump relaxation could be identified by its field dependence. The intrabond motion is frozen out below $T_{c}$ and is fast enough above $T_{c}$ to have no field dependence. Thus it was clearly identified by its temperature-dependent contributions to relaxation. Additional confirmation of the correctness of assignments to intrabond and interbond relaxation was obtained by measuring separately the one-quantum and two-quantum transition probabilities $P_{1}$ and $P_{2}$ using suitable pulse sequences applied to the quadrupolarsplit deuteron NMR lines. ${ }^{16}$

The next step in the analysis was to make use of the information which is available on two of the three contributions to deuteron relaxation (interbond jumps, intrabond jumps, and spin diffusion to paramagnetic impurities) in order to obtain an experimental value for the third. The evaluation of the interbond contribution requires only a measurement of the interbond jump time $T_{X Y}$ since the efg tensor in the $\mathrm{X}$ and $\mathrm{Y}$ bonds is already known. The measurement is accomplished by applying a suitable sequence of NMR pulses and observing the results. The value obtained is $T_{X Y}=0.015 \mathrm{sec}$. at $70^{\circ} \mathrm{C}$. The contribution of spin diffusion to deuteron relaxation was estimated from measurements of Eric Jones, graduate student, on the phosphorus and residual proton relaxation in the same crystal. The remaining contribution must be due to intrabond jumps. Its dependence on temperature yields an activation energy for intrabond motion of $0.078 \mathrm{eV}$. Further support for the model, and in particular for the intrabond contribution, is obtained by turning the problem around; i.e., using the measured activation energy in the theoretical expressions for $O_{1}$ and $P_{2}$ and calculating the change in the efg tensor due to intrabond jumps from the rate measurements, and over as wide a temperature range as possible. A temperature independent result is obtained as well as a reasonable consistency of all of the data. Since the intrabond jumps provide the mechanism for effective motion of Takagi $\mathrm{DPO}_{4}$ and $\mathrm{D}_{3} \mathrm{PO}_{4}$ groups, ${ }^{17}$ these measurements may be regarded as the first experimental proof of their existence.

Even with Takagi's modification ${ }^{17}$ to Slater's statistical theory ${ }^{18}$ for $\mathrm{KH}_{2} \mathrm{PO}_{4}$, the predicted spontaneous polarization curve did not fit experiment very well. At this point the transition was still believed to be second order, ${ }^{19}$ and so the disagreement which concerns us here is only with the shape of the curve. Schmidt decided to introduce a long-range interaction into the Slater-Takagi theory, unaware that Senko had already done so. ${ }^{20}$ With considerable theoretical assistance from Uehling and Silsbee, and with the computer expertise of the latter, this theory (often called the SUS theory) was worked out in detail. ${ }^{21}$ Perhaps it should better be called the Slater-Takagi-Senko theory, but SUS carried out its predictions more thoroughly than Senko did. In particular, it was noted that if the long-range interaction is strong enough the transition becomes first-order. As mentioned above (see note 19), the first-order character of the transition was subsequently observed. Furthermore, its possibility provided the basis for Schmidt's prediction ${ }^{22}$ of a pressure-induced tricritical point in $\mathrm{KH}_{2} \mathrm{PO}_{4}$, which was later experimentally confirmed by his group at Montana State University. ${ }^{23,24}$

On the completion of his doctoral dissertation ${ }^{25}$ in 1961 Schmidt joined the Faculty of Valparaiso University, Valparaiso, Indiana where he remained for 
three years before moving on to Montana State University, Bozeman, Montana. The work on protonic conductivity in hydrogen bonded systems which he started in Valparaiso continued and expanded to include dielectric, optical and nuclear magnetic resonance studies. He created a new center of solid state research and an independent program of studies neatly paralleling the work in progress in UW Physics and UW Engineering. Together with his students Schmidt has investigated several of the more unusual ferroelectric and non-ferroelectric solids; as for example, lithium hydrazinium sulfate and thulium aluminum garnet. He has made contributions to the study of hydrogen dynamics in such well studied materials as ice. ${ }^{26} \mathrm{He}$ has made further contributions to the theory of proton dynamics. An early one was his restudy ${ }^{27}$ of hydrogen intrabond motion in $\mathrm{KD}_{2} \mathrm{PO}_{4}$ following new observations of Bjorkstam on the nature of the phase transition. The work of his group on lithium hydrazinium sulfate which demonstrated that this solid was not ferroelectric as previously believed prompted a theoretical study based on the biased one-dimensional stochastic Ising Model. ${ }^{28} \mathrm{~A}$ theoretical investigation ${ }^{29}$ based on a model of solitons propagating in a chain of harmonically coupled particles lying in double minimum potential wells sought to explain cluster growth and decay near the phase transition in hydrogen bonded systems. A chapter in a book $^{30}$ on hydrogen bonds summarized much of the knowledge up to about 1975.

Returning to the work in the UW Physics laboratory, we note that there were two other students, Eric D. Jones and Harold P. Mahon, who completed dissertations at about the same time as Schmidt. Neither of these dealt specifically with the properties of ferroelectrics, but the motivation for one, and the techniques used in the other, provided a certain degree of unity. Spin diffusion relaxation was still regarded as a poorly understood subject and Jones undertook a further investigation. ${ }^{31} \mathrm{He}$ looked specifically for the transition from the spin diffusion limited case to the rapid diffusion case, and he found that in the phosphates the relaxation is spin diffusion limited at all temperatures down to at least $4.2^{\circ} \mathrm{K}$. In the arsenates the relaxation of nuclei with spin $\frac{1}{2}$ was shown to be a combination of spin diffusion and interaction with a rapidly relaxing arsenic nucleus. A consistent interpretation in terms of arsenic level structure that changes drastically on going through $T_{c}$ was given.

Mahon studied spin transitions in ionic crystals which are a consequence of a single phonon interaction between a mode of lattice vibration and a nucleus possessing a quadrupole moment. The objective was to measure the strength of the coupling. In order to make a quantitative measurement one must know simultaneously both the acoustic energy in the crystal and the strength of the nuclear resonance signal. There are many experimental procedures for doing this, and numerous experiments had already been performed, most of them subject to criticism. Mahon ${ }^{32}$ developed another technique which appeared to be an improvement, and he measured the so-called enhancement factor in $\mathrm{NaCl}$, an important parameter of the theory. The principal relevance of this work to this report on ferroelectrics is that the experience gained in this experiment probably had something to do with a new approach to the study of ferroelectrics, the description of which will be given later in this report.

Meanwhile, a development in international relations which was later to have a profound effect on the research in each of the three local groups was occurring. 
We had become aware of the research which was being done under the direction of Robert Blinc in the Jozef Stefan Institute in Ljubljana, Yugoslavia. We discovered that, both in its experimental and theoretical aspects, the investigations in his group and in ours were closely parallel. Exchanges of papers and some correspondence followed. In 1963 Blinc extended an invitation to Uehling to give lectures on hydrogen bond dynamics at the IXth Yugoslavia Summer School in Theoretical Physics in Hercegovina, Yugoslavia. From that time until the present day there has been a continuous collaboration between us. With the passage of time this collaboration has involved the Bjorkstam and Schmidt groups as well as the original UW Physics group. The three of us have made numerous trips to Ljubljana with some of the visits extending over periods of several months. Blinc has been a visitor in both Seattle and Bozeman. On two occasions his stays in Seattle extended over several months as a visiting professor in the department of Physics. Other members of the Ljubljana group have spent periods of up to a year with us. There have also been students exchanges, usually at the post-doctoral level. Several papers ${ }^{33}$ may be cited as examples of our collaboration in research, and attention is directed in particular to two of them. The paper of Blinc and Bjorkstam gives the results of a first measurement of the Slater energy parameter and demonstrated for the first time the cluster (central mode) effects which continue to be of interest to this time. The paper of Blinc and Svetina relates to the SUS theory but approaches the problem from another point of view, and through the inclusion of tunneling (not present in SUS) and a more systematic treatment of long range forces achieves improved agreement between theory and experiment. It is shown that the SUS results are contained within the improved theory.

Four lines of research at UW Physics remain to be described. We begin with the work of Eiichi Fukushima on spin diffusion relaxation, our final effort to obtain a better understanding of this process. He did both an experimental and a theoretical study ${ }^{34}$ The measurements were on rare-earth-doped $\mathrm{CaF}_{2}$. Both the negligible and non-negligible ion density cases were considered, and in the negligible ion density case he studied the three regimes: (1) diffusion limited relaxation; (2) rapid diffusion relaxation; and (3) an intermediate region for which a computer solution was provided by H. B. Silsbee. He also made a study of the transition at small $t$ between the initial linear and the final exponential decays.

Next we mention the work of Jon F. Soest on the proton and deuteron relaxation in antiferroelectric $\mathrm{NH}_{4} \mathrm{H}_{2} \mathrm{PO}_{4}$ and its deuterated isomorph. ${ }^{35}$ Principal interest here lies in the deuterated crystal in which the quadrupole interaction allows separate identification of hydrogen bond and ammonium deuterons except at "cross-over" where lines coincide. Temperature, field and orientation dependence studies permit a separation of the various contributions leading to a measurement of interbond and intrabond activation energies and interbond jump time. Analysis of the spectra at and below the transition temperature provided new results on the ordering of deuterons within hydrogen bonds responsible for the antiferroelectric structure.

Cross-over is another subject to which we keep returning, and we mention it again in the context of a comprehensive investigation made by Wendland 
Beezhold ${ }^{7,36}$ to which brief reference has already been made. Beezhold calculated the cross-over pattern for $\mathrm{KH}_{2} \mathrm{AsO}_{4}$ using a model of the efg tensor at the As site which is suggested by the crystal structure in the ordered state, and he measured the proton relaxation rate as a function of crystal orientation in the magnetic field at several temperatures below the Curie point. These patterns consisting of several minima in $T_{1}$ as a function of angle, are extremely complicated, but in every detail good agreement between the theoretical and experimental results was obtained. An experimental value of the electric quadrupole coupling constant and the principal axes orientation of the efg tensor are obtained. Also, the absolute value of the proton relaxation rate was explained in terms of dipolar coupling between protons and the rapidly relaxing As nuclei together with strain broadening in the As transitions.

A final contribution from UW Physics carries us back to the first work of Bjorkstam and Schmidt and their development of the microscopic concepts of deuteron interbond and intrabond motion. This work begins phenomenologically with the measurement of elastic constants as determined by sound velocity measurements. It is the work of Eliyahu Litov ${ }^{37}$ who performed the experiments and contributed much to the establishment of connections between macroscopic and microscopic concepts. From measurements on acoustic shear waves in $\mathrm{KD}_{2} \mathrm{PO}_{4}$ propagating and polarized in the plane perpendicular to the spontaneous polarization axis one obtains numerical values of attenuation and elastic constants. Phenomenological equations then permit a determination of the polarization relaxation time and the susceptibility. One virtue of the experiment is that the data are clean and easily reproduced. A good determination is made of the Curie-Weiss constant, and the first order character of the transition is evident. The next step is the connection with microscopic concepts. It is established here through the use of SUS theory. As a consequence of this connection some further insights into the theory may have been established. Introducing previously determined values of the Slater-Takagi energy parameters and making some reasonable choices of theoretical parameters not yet determined, theoretical plots of polarization relaxation time in good agreement with experimental results are obtained. Theoretical aspects of this study, including also an extension to non-ferroelectric phase transitions, were described by one of the authors at a summer school in Yugoslavia in $1971 .^{38}$

Litov has continued his research along lines initiated in this thesis research, first at the Massachusetts Institute of Technology, Cambridge, Massachusetts, and then at Bar-Ilan University, Ramat-Gan, Israel. A theoretical paper ${ }^{39}$ on transverse susceptibility in $\mathrm{KH}_{2} \mathrm{PO}_{4}$ type crystals which makes explicit use of the concept of biased hydrogen intrabond motion provided theoretical results which agree moderately well with the rather meagre available transverse susceptibility data. This work became the starting point for further studies by Litov and his co-workers in Israel.

\section{REFERENCES}

1. J. L. Bjorkstam and E. A. Uehling, Bull. Am. Phys. Soc. 2, 383 (1957).

2. This crystal was kindly supplied by H. Jaffe of the Clevite Research Center. In later stages of the 
research additional cyrstals (deuterated and undeuterated and including others isomorphic to $\mathrm{KH}_{2} \mathrm{PO}_{4}$ ) were obtained from Werner Känzig of Cornell University, who, in additon to supplying us with crystals, became a most valuable source of information directly relevant to out research. At a still later stage crystals grown in our laboratory, often for the specific purpose of achieving a well-controlled paramagnetic ion impurity content, were used. The crystal growing program was initiated and developed by a beginning graduate student, Wendland Beezhold.

3. J. L. Bjorkstam, Ph.D. Thesis, University of Washington (1958).

4. J. L. Bjorkstam and E. A. Uehling, Phys. Rev. 114, 961 (1959).

5. J. L. Bjorkstam and E. A. Uehling, Bull Am. Phys. Soc. 3, 166 and 318 (1958).

6. J. L. Bjorkstam, Bull. Am. Phys. Soc. 7, 464 (1963); P. Kelly, M.S. Thesis, University of Washington (1966).

7. Wendland Beezhold and E. A. Uehling, Phys. Rev. 175, 624 (1968).

8. J. L. Bjorkstam and J. H. Willmorth, Proc. VIIth AMPERE Colloquium on Magnetic Resonance and Relaxation, ed. R. Blinc (North-Holland, Amsterdam, 1976), pp. 728-738; J. L. Bjorkstam, J. Phys. Soc. Japan (Supplement) 28, 10 (1970).

9. J. L. Bjorkstam, E. D. Jones, H. B. Silsbee and E. A. Uehling, J. Chem. Phys. 37, 469 (1962); R. Blinc and J. L. Bjorkstam, Phys. Rev. Letters 23, 788 (1969); G. L. Adriaenssens and J. L. Bjorkstam, J. Chem. Phys. 55, 1137 (1971); G. L. Adriaenssens, J. L. Bjorkstam and J. Aikins, J. Mag. Res. 7, 99 (1972).

10. J. L. Bjorkstam and R. E. Oettel, Phys. Rev. 159, 427 (1967); J. L. Bjorkstam and R. E. Oettel, Proc. of the Intl. Meeting on Ferroelectricity (Czechoslovak Academy of Sciences, Prague, 1966), eds. V. Dvorak, A. Fouskova and P. Glogar, vol. II, pp. 91-96; J. L. Bjorkstam and R. E. Oettel, The Trend in Engineering at the University of Washington, vol. 17, no. 4, 8 (Oct. 1965).

11. W. R. Elliott and J. L. Bjorkstam, J. Phys. Chem. Solids 25, 1273 (1964).

12. R. L. Himbarger and J. L. Bjorkstam, Appl. Phys. Letters 3, 109 (1963).

13. R. L. Mayer and J. L. Bjorkstam, J. Phys. Chem. Solids 23, 619 (1962); G. L. Adriaenssens and J. L. Bjorkstam, Phys. Stat. Solidi A 18, 129 (1973).

14. L. Onsager to E. A. Uehling, private communication, Dec. 18, 1959.

15. L. Onsager to E. A. Uehling, private communication, Jan. 18, 1960.

16. V. H. Schmidt and E. A. Uehling, Phys. Rev. 126, 447 (1962).

17. Y. Takagi, J. Phys. Soc. Japan 3, 271 and 273 (1948).

18. J. C. Slater, J. Chem. Phys. 9, 16 (1941).

19. The first order character of the transition was demonstrated several years later when Bjorkstam observed the simultaneous presence of both high and low temperature spectra over an interval of approximately $1^{\circ} \mathrm{C}$ near $T_{c}$ in an experiment which also displayed the intrabond deuteron motion above $T_{c}$. See J. L. Bjorkstam, Phys. Rev. 153, 599 (1967).

20. M. E. Senko, Phys. Rev. 121, 1599 (1961).

21. H. B. Silsbee, E. A. Uehling and V. H. Schmidt, Phys. Rev. A 133, 165 (1964).

22. V. H. Schmidt, Bull. Am. Phys. 19, 649 (1974).

23. V. H. Schmidt, A. B. Western and A. G. Baker, Phys. Rev. Letters 37, 839 (1976).

24. A. B. Western, A. G. Baker, C. R. Bacon, and V. H. Schmidt, Phys. Rev. B 17, 4461 (1978).

25. V. H. Schmidt, Ph.D. Thesis, University of Washington (1961).

26. Deuteron NMR Relaxation in $\mathrm{D}_{2} \mathrm{O}$ Ice Ih. Physics and Chemistry of Ice (Royal Society of Canada, Ottawa 1973), eds. S. J. Jones and L. W. Gold.

27. V. H. Schmidt, Phys. Rev. 164, 749 (1967).

28. V. H. Schmidt, Journ. Math. Phys. 12, 992 (1971).

29. V. H. Schmidt, Phys. Rev. B 20, 4397 (1979).

30. V. H. Schmidt, a chapter on Ferroelectric Hydrogen-bonded Systems in Recent Progress in Hydrogen Bonds (North Holland Publishing Company, Amsterdam) 1975.

31. Eric Daniel Jones, Ph.D. Thesis, University of Washington (1962).

32. Harold Patterson Mahon, Jr., Ph.D. Thesis, University of Washington (1961).

33. R. Blinc and J. L. Bjorkstam, Phys. Rev. Letters 23, 788 (1969); R. Blinc and E. A. Uehling, Phys. Letters 20, 337 (1966); R. Blinc and S. Svetina, Phys. Rev. 147, 423 and 430 (1966).

34. Eiichi Fukushima, Ph.D. Thesis, University of Washington (1967); Eiichi Fukushima and E. A. Uehling, Phys. Rev. 173, 366 (1968).

35. Jon Frederick Soest, Ph.D. Thesis, University of Washington (1967).

36. Wendland Beezhold, Ph.D. Thesis, University of Washington (1969).

37. Eliyahu Litov, Ph.D. Thesis, University of Washington (1968); E. Litov and E. A. Uehling, Phys. Rev. Letters 21, 809 (1968); E. Litov and E. A. Uehling, Phys. Rev. B 1, 3713 (1970).

38. E. A. Uehling, Ampere International Summer School II, Pulsed Magnetic and Optical Resonance, Basko polje, Yugoslavia (1971), p. 279.

39. S. Havlin, E. Litov, and E. A. Uehling, Phys. Rev. B 9, 1024 (1974). 